\title{
Six-months assessment of a hand prosthesis with intraneural tactile feedback
}

F.M. Petrini ${ }^{1,{ }^{*}}$, G. Valle ${ }^{1,3,{ }^{*}}$, I.Strauss ${ }^{1,3,{ }^{*},}$, G. Granata ${ }^{4,5,{ }^{*}}$, R. Di Iorio ${ }^{4,5}$, E. D'Anna ${ }^{1}$, P. Čvančara ${ }^{6}$, M. Mueller $^{6}$, J. Carpaneto ${ }^{3}$, F. Clemente ${ }^{3}$, M. Controzzi ${ }^{3}$, L. Bisoni ${ }^{7}$, C. Carboni ${ }^{7}$, M. Barbaro ${ }^{7}$, F. Iodice ${ }^{4,5}$, D.

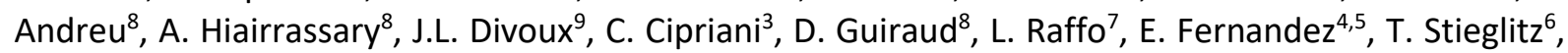
S. Raspopovic ${ }^{2,+}$, P.M. Rossini ${ }^{4,5,+}$, S. Micera ${ }^{1,3,+}$

${ }^{1}$ Bertarelli Foundation Chair in Translational Neuroengineering, Centre for Neuroprosthetics and Institute of Bioengineering, School of Engineering, École Polytechnique Fédérale de Lausanne (EPFL), Lausanne, Switzerland.

${ }^{2}$ Laboratory for Neuroengineering, Department of Health Sciences and Technology, Institute for Robotics and Intelligent Systems, ETH Zürich (ETH), Zürich, Switzerland

${ }^{3}$ The Biorobotics Institute, Scuola Superiore Sant'Anna, Pisa, Italy.

${ }^{4}$ Institute of Neurology, Catholic University, Rome, Italy.

${ }^{5}$ Department of Geriatrics, Neuroscience and Orthopaedics, Policlinic A. Gemelli FoundationIRCCS, Rome, Italy.

'Laboratory for Biomedical Microtechnology, Department of Microsystems EngineeringIMTEK; Bernstein Center Freiburg and BrainLinks-BrainTools Center University of Freiburg, Freiburg, Germany.

${ }^{7}$ Department of Electrical and Electronic Engineering, Università di Cagliari, Cagliari, Italy.

${ }^{8}$ University of Montpellier, INRIA, CAMIN team, 860 Rue St Priest, 34090 Montpellier, France.

${ }^{9}$ Axonic company, 2720 Route de St Bernard, 06224 Vallauris, France.

*EQUAL CONTRIBUtors AS JUNIOR AUthors

+EquAl CONTRIBUtORS AS SENIOR AUtHORS

CORRESPONDING AUTHORS: SILVESTRO MICERA (SILVESTRO.MICERA@EPFL.CH [SANTANNAPISA.IT]), PAOLO M. ROSSINI (PAOLOMARIA.ROSSINI@POLICLINICOGEMELLI.IT) 


\begin{abstract}
Objective: Hand amputation is a highly disabling event, which significantly affects quality of life. An effective hand replacement can be achieved if the user, in addition to motor functions, is provided with the sensations that are naturally perceived while grasping and moving. Intraneural peripheral electrodes have shown promising results toward the restoration of the sense of touch. However, the long-term usability and clinical relevance of intraneural sensory feedback have not yet been clearly demonstrated.
\end{abstract}

Methods: To this aim, we performed a six months clinical study with three trans-radial amputees who received implants of transverse intrafascicular multichannel electrodes (TIMEs) in their median and ulnar nerves. After calibration, electrical stimulation was delivered through the TIMEs connected to artificial sensors in the digits of a prosthesis to generate sensory feedback, which was then used by the subjects while performing different grasping tasks.

Results: All the subjects, notwithstanding their important clinical differences, reported stimulationinduced sensations from the phantom hand for the whole duration of the trial. They also successfully integrated the sensory feedback into their motor control strategies while performing experimental tests simulating tasks of real life (with and without the support of vision). Finally, they reported a decrement of their phantom limb pain and a general improvement in mood state.

Interpretation: The promising results achieved with all subjects show the feasibility of the use of intraneural stimulation in clinical settings. 


\section{Introduction}

The loss of a hand affects persons's quality of life ${ }^{1}$. From the first manufactured prostheses ${ }^{2}$, more effective clinical solutions have been provided: commercially available hands have been developed ${ }^{3}$, enabling users to control the movement of up to six degrees of freedom. In parallel, a huge effort has been conducted in research to provide more dexterous artificial devices ${ }^{3}$, along with decoding methods allowing their control ${ }^{4,5}$; and, as requested by the final users ${ }^{6,7}$, to re-establish the sensory flow of information between the hand prosthesis and the central nervous system, through noninvasive and invasive approaches ${ }^{8-21}$.

Efforts have been dedicated to the use of implantable peripheral interfaces to elicit sensations ${ }^{14-20}$. After the pioneering studies with LIFEs ${ }^{14}$, three interfaces have been used with human subjects: epineural (cuff ${ }^{15-17}$ and FINE $^{15,16}$ ), penetrating (Utah array ${ }^{18,19}$ ), and intraneural (TIME ${ }^{20}$ and LIFE $^{21}$ ) electrodes.

Tyler's group showed that FINEs can be implanted for more than 48 months ${ }^{15,16}$ in two upper-limb amputees allowing them to pull the stem from a cherry ${ }^{15}$. Notwithstanding preliminary encouraging results ${ }^{22}$, there is still no evidence that FINE-induced stimulation can be used to modulate some levels of the prosthesis grasping force, priority for amputees, and one of the reasons such devices are abandoned ${ }^{6,7,23}$. Moreover, the quality of the elicited sensations was little differentiated (mostly paraesthesia was reported ${ }^{15}$ ).

Utah arrays ${ }^{18,19}$ have shown to evoke percepts of more varied quality (tingle, vibration, pressure) in upper-limb amputees but with poor reproducibility in time. In fact, about $80 \%$ of the sensations evoked in two subjects, changed location or type over a period of five weeks.

Finally, TIMEs ${ }^{24}$ were transversally implanted into the residual nerves of an amputee for a period limited to 30 days by EU medical device regulations. During this experiment, TIMEs have been used to restore several features of the sense of touch including sophisticated ones such as the ability to control grasping force, to discriminate different textures, along with objects compliance and shape ${ }^{20,25}$.

To date, TIMEs may represent an interesting trade-off between the other two solutions, reducing their specific limitations in terms of sensations quality (FINEs) and stability (Utah arrays), and allowing the restoration of features such as prosthesis grasping force control. However, the sole study investigating it in humans, was conducted on a single subject for only a month and with no detailed characterization of all the evoked sensations. Moreover, it is not clear $^{26}$ yet to what extent sensory feedback elicited by all these interfaces (not only TIMEs) can improve motor control performance in simulated ecological conditions ${ }^{27}$ (e.g., handling fragile objects when users can exploit both visual and tactile feedback). Finally, even if anecdotally reported that direct nerve stimulation induces phantom limb pain relief ${ }^{15,21}$, a more systematic characterization of the phenomenon has never been conducted.

In order to address these three issues, we conducted a six-months clinical study with three trans-radial amputees implanted with four TIMEs in the residual median and ulnar nerves. We intentionally recruited subjects with different clinical conditions (long or short time since the amputation, affected or not by phantom pain) to investigate the exploitability of our approach for a variegated population of trans-radial amputees.

After characterizing the subjects' response to the intraneural stimulation, we investigated the effects of sensory feedback on the performance of the subjects during different reaching and grasping tasks accomplished with the support of vision (in ecological conditions), and without it. Finally, we assessed the effect of intraneural stimulation on phantom limb pain as well as on mood states during the period of study.

\section{Materials and Methods}

\section{Patient recruitment}

Three left trans-radial amputees were included in the study (Table 1). The first subject (FNG) was a 37year-old male, left-handed, who had a traumatic trans-radial amputation of the distal two-thirds of the 
left forearm 2 years before the enrollment in the trial. At the time of the trial he was affected by strong phantom limb pain and very frequent non-painful phantom sensations. In addition to the amputation and the consequent phantom limb syndrome the clinical condition of the patient was unremarkable. The second subject (ALM) was a 48-year-old female, right-handed, with a traumatic transradial amputation of the distal third of the left forearm, occurred 23 years before the study. She was free of phantom pain and experienced very mild and infrequent spontaneous non-painful phantom sensations.

The third subject (LRP) was a right-handed, 53-year-old female trans-radial (distal two-thirds of the left forearm) amputee. The amputation occurred in December 2015, following a traumatic accident at work. She was affected by phantom pain.

Ethical approval was obtained from the Institutional Ethics Committees of Policlinic A. Gemelli-IRCCS at the Catholic University, Rome, Italy, where the surgery was performed. The protocol was also approved by the Italian Ministry of Health, division for experimental devices. All the subjects signed the informed consent. During the entire duration of our study, all experiments were conducted in accordance with EU guidelines and regulations.

\section{Surgical procedures}

Four TIMEs (14 active sites each) were implanted in the median and ulnar nerves of FNG on November $14^{\text {th }} 2015$, of ALM on June $24^{\text {th }} 2016$, and of LRP on June $24^{\text {th }} 2017$ (Fig. 1A and Supp. Video 1 ). The explant of the electrodes was executed on June $11^{\text {th }} 2016$, on December $17^{\text {th }} 2016$, and on December $16^{\text {th }} 2017$, for FNG, ALM, and LRP respectively.

The surgical approach used to implant TIMEs is extensively reported elsewhere ${ }^{20}$. Briefly, during general anesthesia, through a $15 \mathrm{~cm}$-long skin incision on the left arm, the median and ulnar nerves were exposed to implant a proximal and a distal TIME in each nerve (Fig. 1A). The microelectrodes and a segment of their cables were drawn through four small skin incisions into proximity with the exposed nerve, two laterally and two medially to the center of the main surgical cut. The cable segments were located in subcutaneous pockets, externalized (and secured with sutures and subcutaneous strain release loops) in order to be available for the transcutaneous connection with a neural stimulator. Then, using an operating microscope (Zeiss, Pentero), the single microelectrode was implanted transversally within the nerve fascicles. Appropriate microsutures secured the nerve implant. This implantation procedure lasted 11 hours for FND, 8 hours for ALM, and 11 hours for LRP. The impedance of the electrodes active sites was monitored intraoperatively, to verify the integrity and functionality of the implant. After 180 days, the microelectrodes were removed under an operating microscope in accordance with the protocol and the obtained permissions.

A clinical and electrophysiological follow up was executed at four months for ALM, ten months for FNG, and three months for LRP.

\section{Perceived sensations characterization}

Regarding the subjects' response to the intraneural stimulation (sensation characterization or mapping procedure), the prosthesis was not worn, and the patient was connected only to the neurostimulator using the transcutaneous cables of the implanted TIMEs. The stimulation was triggered by the experimenter using a custom-made software (Matlab, R2016b, The Mathworks, US). The patient was blind to the stimulation configuration delivered. We injected trains of biphasic charge-balanced cathodic-first pulses of increasing amplitude from $10 \mu \mathrm{A}$ to $980 \mu \mathrm{A}$ (steps of $10 \mu \mathrm{A}$ ), and fixed pulse width (chosen in the range $10 \mu$ s to $120 \mu$ s depending on the active site) and frequency ( $50 \mathrm{~Hz}$ as in our previous study $y^{20}$ ). Pulse trains of 2 seconds duration were delivered with an interval between trains of 2 seconds. The same set of stimulation parameters was delivered three times per active site. Hence, for example, per each active site the sensory threshold was determined as average of the three minimum values of charge (one per repetition, i.e. 2-s train of current pulses) at which the subject reported a percept.

In each of these trains of increasing amplitude, patients had to press a button to stop the stimulation ramp when and whether they perceived a sensation. Then, they had to report using a custom-made 
graphical user interface (Matlab, R2016b, The Mathworks, US) the sensation properties (location, type, quality and intensity). The patients could select a word from a list (similar to the one prepared by Kim and colleagues ${ }^{28}$ ) to describe the evoked sensation, but they could also add a new word if needed.

At the end of this procedure, a map of the sensations type, location, extent and intensity (including the sensory threshold) referred to the correspondent active sites was obtained and used for the calibration of the sensory feedback restoration system.

The sensation characterization was performed once per week or every two weeks, according to the availability of the subjects (if they were spending the whole week at the clinical site for performing the tests or only part of it). In particular, FNG and ALM did it weekly, while LRP every two weeks.

Finally, we assessed the stability of the elicited sensation on the phantom hand over time as change of location. In particular, we considered nine location clusters on the phantom hand (five fingers and four palm areas). A sensation was considered stable when, from one week to the other, an elicited sensation was perceived in the same cluster. We did not consider stump percepts in this analysis, because their stability was not of interest for the study. Conversely, we studied the occurrence of stump sensations over time.

\section{Electrode characterization}

For the characterization of the electrode-nerve impedance and the subjects' response to intraneural stimulation we delivered trains of cathodic-first, biphasic and symmetric square-shaped, currentcontrolled stimulation pulses of variable intensity, duration, and frequency, through a dedicated software controlling an external electrical neural stimulator. The impedance was computed as the ratio between the potential difference between the selected active site and the reference electrode (both, left and right one of the electrode) at the end of the cathodic pulse phase. The potential resulted from the average of 5 pulses with an amplitude of $20 \mu \mathrm{A}$ and a width of $300 \mu \mathrm{s}$, repeated at a frequency of $1 \mathrm{~Hz}$. Electrodes were considered still working if their impedance was below $100 \mathrm{kOhm}$.

\section{Prosthesis movement control}

The subjects controlled a research hand prosthesis (IH2 Azzurra, Prensilia) using the residual muscle activity of their forearm residuum. In total two pairs of disposable surface electrodes $(\mathrm{Ag} / \mathrm{AgCl}, 24 \mathrm{~mm}$ diameter, $20 \mathrm{~mm}$ inter-electrode distance) were placed on the digit flexor and the extensor extrinsic muscles. The signals were sampled at $2 \mathrm{kHz}$ and digitally filtered using a $4^{\text {th }}$ order pass-band (15-375 $\mathrm{Hz}$ ) Butterworth IIR filter, and a notch filter to remove the $50 \mathrm{~Hz}$ power line interference (through Grapevine system, Ripple, LLC, USA). A robust three-state (open $=-1$, close $=1$, rest $=0$ ) k-Nearest Neighbor $\left(\mathrm{kNN}, \mathrm{k}=3\right.$ ) classifier with a decision-based velocity ramp ${ }^{29}$ was used to control the hand. The classifier was fed with the waveform length (WL) of each EMG channel, computed over a window of $100 \mathrm{~ms}$, and allowed a gated-ramp control ${ }^{30}$ of the speed of the robotic hand movements, as in the following relation:

$$
\begin{array}{ll}
V_{\text {hand }} \leftarrow V_{\text {hand }}+A^{*} \text { Class output; } & \text { when } \text { Class }_{\text {output }}=-1,1 ; \\
V_{\text {hand }} \leftarrow 0 ; & \text { when Class output }=0 ;
\end{array}
$$

where $A$ is a proportional factor that can be controlled by the experimenter, Class output $_{\text {is }}$ is the output of the classifier, and $V_{\text {hand }}$ is a number in the range between 0 and 511, that controls the velocity of the motors of the robotic hand. When $V_{\text {hand }}=1$ the angular velocity of the robotic hand is $0.297 \mathrm{deg} / \mathrm{sec}$, when $V_{\text {hand }}=511$ the angular velocity of the robotic hand is $511^{*} 0.297 \mathrm{deg} / \mathrm{sec}=151.76 \mathrm{deg} / \mathrm{sec}$.

The classifier required a training session every time the surface EMG electrodes were newly placed. During the training the user was asked to relax (one time for $2 \mathrm{~s}$ ), then open (one time for $2 \mathrm{~s}$ ) and finally close (one time for $2 \mathrm{~s}$ ) the phantom hand. The resulting EMG activity was recorded and manually separated into three classes basing on the WL.

\section{Prosthesis Sensory Feedback}

Two force sensors embedded in the little and index fingers of the prosthesis were used as control 
inputs for the intraneural stimulation of two active sites in the sensory innervation territories of the median and ulnar nerves. In particular, the amplitude of biphasic, symmetric, cathodic-first and rectangular charge-balanced pulses was modulated according to the following linear relationship:

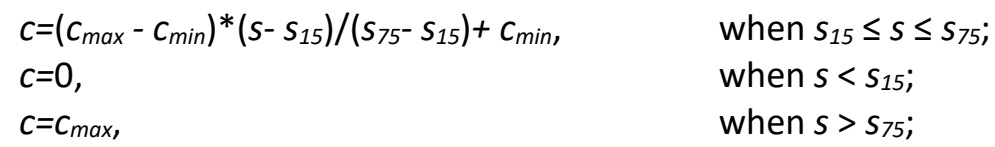

where:

$c$ is the amplitude of stimulation current, $s$ is the sensor readout, $\mathrm{s} 15$ and $\mathrm{s} 75$ represent $15 \%$ and $75 \%$, respectively, of the maximum range of the sensor readout, which characterize, respectively, the contact threshold of the robotic hand and a value tuned to exploit the full range of sensations for all objects. $\mathrm{C}_{\min }$ and $\mathrm{C}_{\max }$ are the stimulation current amplitudes that elicited, respectively, the minimum and maximum (i.e., below pain threshold) touch sensations as reported by the subject according to the last sensation characterization procedure. The frequency of the stimulation is $50 \mathrm{~Hz}^{20}$. The overall control scheme is provided in Fig. $1 \mathrm{~A}$.

We used two active sites for the development of the bidirectional prosthesis, one in the sensory innervation territories of the median nerve and one of the ulnar nerve, eliciting sensations respectively in an area of the phantom hand that was the closest to the prosthesis index finger and in an area closest to the little finger. This was chosen to maximize the somatotopy of the phantom sensations during prosthesis exploitation.

\section{Neurostimulators}

Three neural stimulators were used to inject currents into the nerves of the two subjects by means of TIME electrodes: STIMEP, EARNEST and Grapevine Neural Interface System.

STIMEP (University of Montpellier and Axonic) is a wearable neural stimulator based on a generic neural stimulation architecture ${ }^{31,32}$. It allows the control of 64 poles of implanted electrodes, divided in 4 ports of 16 poles each. Therefore, up to 4 TIME electrodes can be driven in real-time (pulse-width, intensity, frequency). Each port addressing each TIME electrode includes 14 capacitively coupled active outputs (channels) and 2 non-capacitively coupled references. STIMEP further embeds safety procedures (charge limit) and impedance measurements. This stimulator was used for all the tests of FNG.

EARNEST (University of Cagliari) is a prototypical, wearable, complete embedded platform for neural prosthetic applications ${ }^{33}$. It allows to simultaneously control and use up to 4 implantable multi-site (16 channels) electrodes, placed on the same subject, for a total of 64 stimulation/recording channels. The device allows recording of neural signals and, at the same time, provides electrical stimulation fully programmable in terms of amplitude, duration, shape and frequency. It embeds also a recording module for electromyographic (EMG) signals from 4 differential surface electrodes. This stimulator was used on ALM for Sensation characterization, Force Control Test and Blindfolded Sensory Blocks Test. The Grapevine Neural Interface System (Ripple, LLC) is a commercial device that can be used for the recording of neurophysiological data and for delivering current-controlled stimulation through up to 32 high-impedance microelectrodes. This system was used on ALM for the Sensation characterization, Force Control Test, Virtual Eggs Test, and for the whole trial with LRP.

\section{Validation Tasks}

In order to verify whether sensory feedback could allow subjects to improve performance in the prosthesis movement control, three functional tasks were used: Virtual Eggs Test $\left(\mathrm{VET}^{34}\right)$, Blindfolded Sensory Blocks Test $\left(\mathrm{BSBT}^{12}\right)$, Force Control Test $\left(\mathrm{FCT}^{20}\right)$.

First of all, in order to assess whether intraneural stimulation in addition to vision could improve motor control we asked the subjects to perform a test resembling the realistic task of transferring fragile objects (such as eggs), namely the Virtual Eggs Test (VET). The VET is a recently proposed test for sensorimotor assessment ${ }^{34}$. In brief, it is a modification of the well-known box and blocks test ${ }^{35}$ except 
that fragile/breakable blocks (resembling virtual eggs) are used instead of the standard wooden ones. During the VET, the subjects, wearing the prosthesis, are instructed to transfer the fragile blocks presented in front of them from one side to the other over a $15 \mathrm{~cm}$ tall wall as fast as possible and without breaking them. The performance is measured as a percentage, by the number of transferred blocks over the total number of blocks (transferred plus broken) during 1-minute trials, similar to the standard box and blocks test. In this work, the virtual eggs $\left(40 \times 40 \times 40 \mathrm{~mm}^{3}\right.$ blocks, $\sim 80 \mathrm{~g}$ ) exploited a magnetic fuse mechanism, which would collapse/break when grasped with a grip force larger than a specified threshold. The latter was calibrated at a force value that was roughly $50 \%$ larger than the grip force required by the artificial hand to lift it $(1.23 \pm 0,02 \mathrm{~N})$. This value was validated during the tests with FNG (four trials feedback-on and four trials feedback-off). The test is schematized in Fig. 2A and shown in Supp. Video 2. The VET was performed by FNG at month 4 after the surgery, by ALM at months 2 and 3 , and LRP at months 2 and 3 .

Once we assessed the benefit of intraneural sensory feedback in addition to vision during tasks requiring sensibility, we further tested the efficacy of our approach during two additional experiments, in which the volunteers were blindfolded and acoustically isolated: the Blindfold Sensory Blocks Test and the Force Control Test.

In the BSBT subjects, wearing the prosthesis, transferred wooden and unbreakable blocks, during 240 $\mathrm{s}$ sessions, from one side of a table to the other (Fig. 2D and Supp. Video 2). In particular, an operator presented or not objects to the prosthesis, and the subjects had to decide whether he/she had successfully grasped them, and then had to transfer them to the other side of the table. If the participant felt that no object was gripped, he/she was instructed to re-open the hand for the next repetition. The performance was rated through a score: one point was attributed for every successfully transferred block or when the subjects correctly identified that there was not an object in their robotic hand; in any other condition, no points were assigned, and errors were counted. Since the same score was given to the recognition of a grasped object and of a missing object, the number of transfers (hence, the overall score) depended on the time the subjects spent to take a decision about a grasp. The number of object presentation events was balanced with the case in which no object was placed in the prosthesis. This design was put in place to avoid performance discrepancies between trials due to objects seeking. The BSBT was performed by FNG at months 2,3 after the surgery, by ALM at month 2 , and LRP at months 2 .

In the FCT (Fig. 3A), as in Raspopovic et al. ${ }^{20}$, the subjects acoustically shielded and blindfolded were asked to apply 3 levels of grasping force (low, medium and high) on a dynamometer, using (but not wearing) the robotic hand. The prosthesis and the dynamometer were constrained by two presses at a constant position, to guarantee the repeatability of the grasps. The participants were instructed to rely on the sensory information to judge how much force they were applying and to stop when they considered they had reached the desired level (low, medium or high). They had to decide the amount of force required to apply its minimum, medium and maximum level. The patients performed a short familiarization session (approx. 5 minutes), during which they could squeeze the dynamometer with the bidirectional prosthesis exploring the sensory feedback. Then, the experiment was performed. The experimenter asked the requested force level touching the patients' shoulder one (low), two (medium) or three (high) times. Patients reproduced the desired force level, maintaining the level for $2 \mathrm{~s}$, and then they returned to the initial hand position (fully open hand) ready for the next level. The velocity of the hand movement execution was randomly modified ( 3 velocities, by setting $A=1, A=2, A=3$ in (1)) without informing the participants, in order to prevent that they could rely on learned closing times to execute the task. The FCT was performed by FNG at months 2 and 5 after the surgery, by ALM at months 2 and 5 , and LRP at months 2 and 5.

\section{Phantom limb pain and mood states assessment}

FNG and LRP were asked to fill the Neuropathic Pain Symptom Inventory $\left(\left.\mathrm{NPS}\right|^{36}\right.$ ), the Visual analogue scale $\left(\mathrm{VAS}^{37,38}\right)$, and the Profile of Mood States ${ }^{39}$ questionnaires, during 6 weeks before the implant, and each day they received the intraneural stimulation (i.e. every week). ALM, not having any phantom limb syndrome did not fill the questionnaires. 


\section{Statistical analysis}

All data were extracted and processed in Matlab (R2016b, The Mathworks, US). The normality of the data was first checked (one-sample Kolmogorov-Smirnov test). Since none of the data was normally distributed, for the analyses in the paper a two-tailed Friedman's test was used to analyze data and, when needed, a Tukey-Kramer correction for multi-group comparison was applied. Only for performance comparisons over time, i.e. first and last session of VET (Fig. 2), BSBT (Fig. 2), and FCT (Fig. $3)$, a Fisher's exact test was computed. For the comparison of different levels of intensity of sensations, a Kruskal-Wallis test was executed.

Additionally, for the analysis of the force levels (Fig. 3), for each trial, the duration was normalized, and an average force value was computed over a fixed interval (interval from $60 \%$ to $90 \%$ of trial completion). To compute the "performance" score (given as a percentage of correct trials), we first obtained the average force value for each force level, using the method outlined above. Then, we assigned each repetition to the nearest force level. Finally, we computed the performance score as the percentage of repetitions correctly assigned to the right force level.

The number of repetitions per sessions and the number of sessions per test are reported in the figures captions.

\section{Results}

\section{Surgical results}

No side effect related to the surgical procedures was observed. Complications related to the median and ulnar nerves secondary to surgical injury, infection, bleeding or relocation of the electrodes were not observed in FNG and LRP during the trial. ALM had an infection from three of the implants (Staphylococcus Aureus, confirmed by wound swab culture) two weeks after the surgery. During this period one electrode fell out (after seven weeks), while the patient was sleeping. The infection was treated and eradicated with six weeks of combined antibiotic therapy. Nevertheless, also in the case of ALM there were no complications related to the median and ulnar nerve injury at the end of the trial. The follow up excluded long-term complications or loss of function with respect to pre-surgery conditions. The results presented in the rest of the manuscript take into consideration three electrodes for FNG and ALM, and four for LRP. Indeed, as mentioned, ALM lost an electrode after 7 weeks, while for FNG the external connector of one implant completely broke at week 20.

\section{Perceived sensation}

Throughout the whole trial, all the subjects reported sensations elicited by intraneural stimulation on the phantom hand (Fig. 1B). In terms of location, FNG reported sensations in all his phantom digits, while subject ALM felt phantom sensations more on the palm and LRP more on the ulnar territories of innervation of the hand (Fig. 1B). Interestingly, at the beginning of the trial ALM and LRP reported sensations mainly localized at the residuum, while over time most of these perceptions progressively moved to the phantom hand (Fig. 1C).

Along with the trial, the perceptions became very stable: almost $100 \%$ of the active sites for ALM, FNG, and LRP elicited a sensation that maintained the exact same phantom hand location (Fig 1D). ALM and LRP reached very soon this level of stability, while FNG, which reached about $80 \%$ stability at the $4^{\text {th }}$ month, needed all the study period.

A wide range of sensation types (11 different ones) was elicited by the intraneural stimulation (Fig. 1E): as a note, FNG and LRP experienced a feeling of electricity in the phantom hand less than about $10 \%$ of the times, while in ALM such percept was reported in three cases out of four.

Analogously to the location of the elicited sensations, also the minimum charge (threshold) required to elicit a percept reached a plateau at around $20 \mathrm{nC}$ for FNG, $40 \mathrm{nC}$ for ALM, and $60 \mathrm{nC}$ for LRP (Fig. $1 F, G, H)$, respectively. It is interesting to notice that while for the first two subjects, the plateau was reached after a period of adaptation ( 4 and 7 weeks, for FNG and ALM, respectively, $p<0.05$, Fig. 1F,G), 
for LRP the threshold recorded the first week of experiments, was the same until the explant (Fig. $1 \mathrm{H}$ ). All these thresholds were far below the tolerated maximum safe charge injection capacity of the TIMEs, determined to be $120 \mathrm{nC}^{24}$.

Finally, all the subjects reported a direct proportional correlation between the charge of the current injected in the electrodes active sites and the intensity of the evoked sensations (Fig. 4). Conversely, a raise of the injected charge, even if producing a very limited increase of elicited sensations extension, did not affect their location cluster (intended as defined before).

\section{Electrode characterization}

The impedance of the active sites was stable for FNG and LRP at $80 \mathrm{k} \Omega$ and $70 \mathrm{k} \Omega$, respectively, since the beginning of the study, while for ALM changed after the implant in the residual nerves, to stabilize after 3 weeks around a plateau of $40 \mathrm{k} \Omega$ ( $p>0.05$, Fig. 5A,B,C, top). Equally important, we noticed a reduction of around $45 \%$ of usable channels (impedance higher than $100 \mathrm{k} \Omega$ ) from the three studied electrodes towards the end of the study for FNG and ALM (Fig. 5A,B, bottom). Further investigation, though, of the explanted electrodes allowed us to relate this event exclusively to a loss of electrical continuity between the extracorporeal connectors and the transcutaneous cables (Fig. 5D) due to repeated mechanical stress during connection and disconnection of the TIMEs from the stimulator. Indeed, neither the active sites nor the two segmented counter electrodes per TIME showed signs of corrosion due to electrical stimulation or delamination from the substrate and the insulation (Fig. 5E). No alterations of the assembling structures of the ceramic carrier with the thin-film structure, the precision mechanics cables or the cables themselves were observed during optical inspection (Fig. 5E). Wire breakage at the connector site was actually the only failure mode that occurred.

In order to avoid this breakdown, the portion of the electrodes cables of LRP that was solicited because of the continuous connection and disconnection with the external stimulator, was reinforced with an additional layer of silicon. Thanks to this expedient, the four electrodes of LRP had about $90 \%$ of active sites functioning until the end of the study (Fig. 5C, bottom).

\section{Validation tasks}

In the VET, all the subjects transferred a number of unbroken virtual eggs significantly larger when the intraneural feedback was provided with respect to the no-feedback condition ( $57 \%$ vs. $72 \%$ for FNG, $18 \%$ vs. $28 \%$ for ALM, $45 \%$ vs $70 \%$ for $L R P, p<0.05$ ) (Fig. $2 B$ ). Interestingly, all the patients increased the percentage of unbroken and transferred blocks (manual accuracy) when using the sensory feedback condition, maintaining the number of transferred blocks in total (gross manual dexterity).

No significant improvement over time in the number of transferred virtual eggs (overall number or successfully displaced items) was observed (Fig. 2C).

The BSBT was successfully executed by FNG, ALM, and LRP. Indeed, the objects displacement score for both of them was higher than the errors count ( 12 versus 4,24 versus 0 , and 13 versus $1, p<0.05$, respectively for FNG, ALM, and LRP, Fig. 2E). For ALM, remarkably, we did not observe any mistaken trial. As in the VET, even if a positive trend in score was observed over the course of the study, this was not statistically significant (Fig. 2F).

Finally, during the $F C T$, the subjects were able to modulate their grip force at the three different levels at the end of the study ( $p<0.05$ for FNG and $p<0.01$ for ALM and LRP, Fig. 3B,C,D,E). ALM and LRP managed since the beginning to complete the task with a performance of about $83 \%$ and $88 \%$ respectively (Fig. 3C,D,F), which was maintained until the last session of tests $\left(22^{\text {nd }}\right.$ week for ALM and $13^{\text {th }}$ week for LRP, $p>0.05$, Fig. 3F). FNG instead began with a score just above chance level ( $41 \%$, Fig. $3 \mathrm{~F}$ ), but improved by more than $50 \%$, achieving a score of about $65 \%$ in the last trial $\left(23^{\text {rd }}\right.$ week for FNG, p $<0.05$, Fig. 3B,F).

\section{Phantom limb pain and mood states assessment}

Remarkably, during the whole period of provision of the intraneural stimulation, both FNG and LRP experienced a reduction of pain, according to all the rating scales that were provided to them. Indeed, FNG reported a significant decrease of $36 \%$ in the NPSI and $57 \%$ in the VAS ( $p<0.01$, Fig. 6A,B). Similarly, 
the NPSI of LRP decreased from 18 to 5 ( $p<0.05$, Fig. 6D), and her VAS from 6 to 3.2 ( $p<0.01$, Fig. $6 C$ ). Finally, we observed that the mood states of the subjects benefited of the use of the bidirectional prosthesis: e.g., the subjects felt less nervous or miserable at the end of the study (Fig. 6E).

\section{Discussion}

\section{Long-term usability of intraneural electrodes}

We proved for the first time that intraneural interfaces are stable (in terms of elicited sensations and from materials science viewpoint) and functional during chronic implantation in humans to deliver tactile sensory feedback. After the clinical trial, the location and the minimum charge needed to elicit a sensation were stable as well as the impedance of the working active sites, confirming our previous results with animal models ${ }^{40}$. During the whole study period, all the subjects reported sensations from the phantom hand, when intraneural stimulation was provided. These (above all the stability of the location of the sensations) are the most important requirements to guarantee the clinical exploitation of the intraneural sensory feedback restoration device. A slight oscillation of the number of active sites with impedance lower than $100 \mathrm{k} \Omega$ was observed from week to week in FNG, ALM and LRP, probably due to the impedance measuring setup. Also, for subject LRP we observed an increase of the number of active sites with a sensation threshold lower than $120 \mathrm{nC}$. This was maybe due to a minor increase over time of sensitivity of the subject to stimulation.

A great variety of sensation qualities has been elicited both intra- and inter-subjects. Many of these sensations ( $60 \%, \sim 15 \%, \sim 50 \%$, respectively for FNG, ALM, LRP, Fig. 1E) can be associated with the ones that can be perceived with the healthy limb when interacting with objects (e.g. pressure or vibration, which we consider "natural" in this manuscript, i.e. similar to the ones that are perceived with the intact limb). It is worth mentioning that in FNG and LRP we also demonstrated that the intraneural stimuli were able to elicit brain responses in the sensory areas (Somatosensory Evoked Potential) with a shape similar to the one evoked when stimulating sensory fibers in intact nerve ${ }^{41}$. Similar sensation qualities were reported in studies on amputees implanted with penetrating electrodes, however the stability of the percepts obtained with them being low, compared to our results ${ }^{18,19}$. Mostly unnatural percepts (paresthesia) were instead reported with epineural electrodes ${ }^{15}$. This observation makes us hypothesize that the quality of elicited sensations is primarily coded by the types of nerve fibers recruited through neural stimulation. This suggests that electrodes able to target a circumscribed population of fibers (likely of similar type ${ }^{42,43}$ ) may be more suitable to elicit natural sensations: indeed, the simultaneous activation of fibers of different types is not physiologic, since they have their own specific firing rates ${ }^{44}$.

We need also to note that some of the sensations reported by the three subjects were unnatural (e.g. electricity). We believe that new encoding strategies must be identified in the future to elicit more natural sensory feedback. We think that, being sensory feedback one of the requirements of amputees for future prostheses and one of the reasons of abandonment of presently available ones ${ }^{7}$, the quality of the restored sensations will play an important role on device acceptance ${ }^{45,46}$. Neural stimulation should elicit sensory feedback being at the same time efficient (i.e. usable to increase motor control performance) and highly natural, as the naturalness of the feedback plays a pivotal role in prostheses acceptance ${ }^{22}$. More investigations are necessary to test whether a more natural sensory feedback could improve besides acceptance, other factors such as prosthesis control, dexterity or embodiment in amputees.

An interesting phenomenon was reported by subjects ALM and LRP during the study. In their case many sensations moved from the residuum to the phantom hand throughout the weeks. We hypothesize that both subjects had a deeply reorganized connectivity between the central nervous system and the peripheral nerves of the stump, with respect to FNG. In LRP, indeed, the nerve ultrasound, performed before the electrodes implant, showed a reduced cross-sectional area (between 4 and $5 \mathrm{~mm}^{2}$ ) of the median nerve in the tract between the elbow and the proximal third of the arm, compared to the physiological values for median ${ }^{47}$ and ulnar nerves ${ }^{48}$. The surgery confirmed the reduced size of the median nerve, compared to the ulnar one (Fig. 7B). In ALM, the long interval 
between the amputation and the enrollment in the study (more than 20 years) may have induced great and stabilized plastic changes in the paths between the brain and the peripheral nerves ${ }^{49}$. It is known ${ }^{49}$ that in such condition the de-afferented areas of the brain can be "invaded" by the adjacent ones (the forearm and the face, in the case of hand amputees), and that the passive electrical activation of fibers originally directed to the lost body part can generate a sensory perception on these areas and not in the phantom ${ }^{49}$. The six-months stimulation of these fibers, combined with the use of a prosthetic hand, may have induced a partial regression of the abovementioned plastic changes. The other option - not excluding the previous one - is that the continuous stimulation of peripheral nerves (generating a peculiar pattern of sensation) combined with the intensive use of the prosthetic hand may have promoted a process of embodiment of the device (taking advantage of brain plasticity), with a progressive association of the peculiar pattern of sensations with the prosthetic hand itself ${ }^{50}$.

Since the clinical features of the two subjects were very different, we hypothesize that this phenomenon could interest a larger population of amputees, with no restriction. Further studies on this matter are needed.

Also, this phenomenon could be exploited in the future to develop novel therapies of neurorehabilitation, which could involve the combination of neurostimulation with other techniques such as virtual reality ${ }^{37,51}$.

As a side but important note, the results from FNG and ALM clearly confirm that the use of a transcutaneous device makes the overall approach more fragile and prone to stress-induced mechanical failures while connecting and disconnecting electrodes and stimulators. These sites of the connectors have undergone redesign on LRP to increase strength and deliver higher strain relieve during every day handling. This solution provided a great step towards immunity to active sites failure until the end of the study. For future clinical practices, though, the solution may be represented by a fully implantable system, which will avoid any daily connection and disconnection between the electrodes cables and the neural stimulator.

\section{Intraneural stimulation improves prosthesis control performance and mood states, while it reduces phantom limb pain}

Results from BSBT strengthen the evidences, about the benefit of using sensory feedback to complete functional tasks, without any visual or acoustic clue, that were previously shown through a trial with 2 subjects implanted with FINE electrodes ${ }^{52,53}$. In the very same trials, the outcome of the clinical tests executed by the two amputees, seemed to display that very low or no improvement is obtained when sensory feedback is provided when also vision is available.

In this work, notably, with the VET, we demonstrated that the control of the prosthesis in ecological conditions is improved by the sensory feedback. Finally, with the FCT, we reinforced previous findings ${ }^{20}$ of the capability, given by intraneural stimulation, to modulate the grasping force exerted through the prosthesis, which is one of the main users' requirements. The ability of the subjects to modulate the prosthesis grasping force comes probably from the combination of: i) the proportional relation between the charge of the intraneural stimulation and the intensity of the elicited sensation, ii) the independency of the cluster location of the evoked perception on the charge of the intraneural stimulation. These properties come probably from the unique selectivity of intrafascicular electrodes, which allow fibers recruitment in a confined area of a fascicle ${ }^{25}$. To date, the capability of controlling several different levels of prosthesis force through the sensory feedback induced by intraneural stimulation has not been shown with other implantable electrodes. As expected, after the results of the sensation characterization proving the stability of intraneural stimulation over time, the subjects were able to execute the different functional tasks throughout the whole study.

Also, we demonstrated that combining sensory feedback to the control of a myoelectric prosthesis results in significant decrease of phantom limb pain and amelioration of the user's mood. This is a great advantage for users since it would avoid them to undergo additional therapies for pain treatment.

Intraneural stimulation is usable with patients with different clinical conditions

The participants involved in the study presented very different clinical conditions. In more detail, FNG 
had a phantom limb syndrome, which moderately affected his daily activities. He had painful and painless phantom sensations (Fig. 6A,C,E-7A).

Only painful phantom sensations were reported by LRP, but these did not affect her activities of daily leaving. Both LRP and FNG underwent the amputation less than 3 years before the enrollment in the study. ALM did not report any phantom pain and lost the hand more than 20 years before the start of the pilot trial.

The clinical differences of FNG, ALM and LRP could justify some discrepancies in terms of elicited sensations and tasks performance. In fact, FNG, reported a difficulty in clearly identifying percepts evoked by intraneural stimulation because of the continuous presence of spontaneous sensations from the phantom hand. This could explain the reduced performance of FNG compared to ALM and LRP, in the FCT.

Conversely, intraneural stimulation evoked in FNG and LRP sensations that could be associated to the ones perceived by the healthy hand, mostly located over the fingers, while in ALM evoked mostly perceptions of electricity, which rarely extended to the digits. In this case, the long interval from the amputation, and the consequent plastic changes in the central nervous system pathways and relays connected to the stump nerves, could have been the reason of the less natural quality of the sensations and their location perceived by ALM. In the case of LRP, even at the end of the study, the stimulation of the median nerve evoked no sensations on the hand. We hypothesize that the Wallerian degeneration of a consistent contingent of fibers innervating the hand (Fig. 7B) after the amputation was to a point that did not allow to elicit sensations from correspondent areas of the phantom limb (i.e. the phantom fingers).

Outstandingly, independently on the peculiarities of each subject, FNG, ALM, and LRP felt sensations on the phantom hand induced by intraneural stimulation for the whole duration of the trial and exploited these sensations to improve the control of the prosthesis. This suggests that the bidirectional prostheses can be used by subjects of very different clinical conditions, which would allow medical doctors to adopt loose inclusion criteria for the selection of users.

\section{Conclusions}

We believe that our findings support the hypothesis that intraneural stimulation represents a valuable clinical solution to provide sensory feedback to trans-radial amputees and that this approach would be ready to be tested with a larger cohort of patients during long-term clinical trials outside the controlled hospital environment, having established already that in the case of TIMEs the device cable/connector portion has to be replaced by a fully implantable solution. Indeed, we showed that intraneural stimulation represents a solution to amputees' requirements for future prostheses, such as the control of the force grip; improves the control of myoelectric prostheses for the completion of tasks executed with or without the assistance of vision; reduces phantom limb pain; and ameliorates patients' mood state.

\section{Acknowledgments:}

The authors are grateful to the participants who committed six months of their life for the experimentation. Funding: This work was supported by the EU Grant CP-FP-INFSO 224012 (TIME project), the project NEMESIS (Neurocontrolled mechatronic hand prosthesis) funded by the Italian Ministry of Health, the EU Grant FP7-611687 NEBIAS (NEurocontrolled BIdirectional Artificial upper limb and hand prosthesiS), the EU Grant FP7-HEALTH-1602547 EPIONE (Natural sensory feedback for phantom limb pain modulation and therapy). The work was also supported by the Swiss National Science Foundation through the National Centre of Competence in Research (NCCR) Robotics and by the Bertarelli Foundation.

\section{Author contribution:}

F.M.P., G.G, M.C, C.Ci, E.F., T.S., D.G., J.L.D., M.B., L.R., S.R, P.M.R., and S.M contributed to the conception and design of the study. F.M.P., S.R., I.S, G.V, E.D.A, J.C, G.G, R.D.I, F.I, F.C, P.C., M.M., D.A., 
A.H., L.B. and C.Ca. contributed to the acquisition and analysis of data, S.M, F.M.P., G.V, G.G, and I.S contributed to drafting the text and preparing the figures.

\section{Potential Conflicts of Interest:}

S.R., F.M.P., and S.M. hold shares of "Sensars Neuroprosthetics Sarl", a start-up company dealing with commercialization of neurocontrolled artificial limbs. C.Ci., M.C. and F.C. hold shares of "Prensilia", a start-up company commercializing robotic hands and assessment tools. D.G. is author of the W02006027473 A1 patent. The other authors do not have anything to disclose. 


\section{References}

1. Meyer TM. Psychological aspects of mutilating hand injuries. Hand Clin. 2003;19(1):41-49.

2. Tropea P, Mazzoni A, Micera S, Corbo M. Giuliano Vanghetti and the innovation of "cineplastic operations." Neurology 2017;89(15):1627-1632.

3. Belter JT, Segil JL, Dollar AM, Weir RF. Mechanical design and performance specifications of anthropomorphic prosthetic hands: A review. J. Rehabil. Res. Dev. Wash. 2013;50(5):599-618.

4. Farina D, Vujaklija I, Sartori M, et al. Man/machine interface based on the discharge timings of spinal motor neurons after targeted muscle reinnervation. Nat. Biomed. Eng. 2017;1(2):0025.

5. Micera S, Carpaneto J, Raspopovic S. Control of Hand Prostheses Using Peripheral Information. IEEE Rev. Biomed. Eng. 2010;3:48-68.

6. Biddiss EA, Chau TT. Upper limb prosthesis use and abandonment: A survey of the last 25 years. Prosthet. Orthot. Int. 2007;31(3):236-257.

7. Wijk U, Carlsson I. Forearm amputees' views of prosthesis use and sensory feedback. J. Hand Ther. 2015;28(3):269-278.

8. Kaczmarek KA, Webster JG, Bach-y-Rita P, Tompkins WJ. Electrotactile and vibrotactile displays for sensory substitution systems. IEEE Trans. Biomed. Eng. 1991;38(1):1-16.

9. Zhang D, Xu H, Shull PB, et al. Somatotopical feedback versus non-somatotopical feedback for phantom digit sensation on amputees using electrotactile stimulation [Internet]. J. NeuroEngineering Rehabil. 2015;12[cited 2018 Apr 9 ] Available from: https://www.ncbi.nlm.nih.gov/pmc/articles/PMC4416276/

10. Antfolk C, D’Alonzo M, Controzzi M, et al. Artificial Redirection of Sensation From Prosthetic Fingers to the Phantom Hand Map on Transradial Amputees: Vibrotactile Versus Mechanotactile Sensory Feedback. IEEE Trans. Neural Syst. Rehabil. Eng. 2013;21(1):112-120.

11. Chai G, Sui X, Li S, et al. Characterization of evoked tactile sensation in forearm amputees with transcutaneous electrical nerve stimulation. J. Neural Eng. 2015;12(6):066002.

12. D’Anna E, Petrini FM, Artoni F, et al. A somatotopic bidirectional hand prosthesis with transcutaneous electrical nerve stimulation based sensory feedback. Sci. Rep. 2017;7(1):10930.

13. Marasco PD, Kim K, Colgate JE, et al. Robotic touch shifts perception of embodiment to a prosthesis in targeted reinnervation amputees. Brain 2011;134(3):747-758.

14. Horch K, Meek S, Taylor TG, Hutchinson DT. Object Discrimination With an Artificial Hand Using Electrical Stimulation of Peripheral Tactile and Proprioceptive Pathways With Intrafascicular Electrodes. IEEE Trans. Neural Syst. Rehabil. Eng. 2011;19(5):483-489.

15. Tan DW, Schiefer MA, Keith MW, et al. A neural interface provides long-term stable natural touch perception. Sci. Transl. Med. 2014;6(257):257ra138-257ra138.

16. Tan DW, Schiefer MA, Keith MW, et al. Stability and selectivity of a chronic, multi-contact cuff electrode for sensory stimulation in human amputees. J. Neural Eng. 2015;12(2):026002.

17. Ortiz-Catalan M, Hla akansson B, Brla anemark R. An osseointegrated human-machine gateway for longterm sensory feedback and motor control of artificial limbs. Sci. Transl. Med. 2014;6(257):257re6-257re6.

18. Davis TS, Wark HAC, Hutchinson DT, et al. Restoring motor control and sensory feedback in people with upper extremity amputations using arrays of 96 microelectrodes implanted in the median and ulnar nerves. J. Neural Eng. 2016;13(3):036001. 
19. Wendelken S, Page DM, Davis T, et al. Restoration of motor control and proprioceptive and cutaneous sensation in humans with prior upper-limb amputation via multiple Utah Slanted Electrode Arrays (USEAs) implanted in residual peripheral arm nerves [Internet]. J. NeuroEngineering Rehabil. 2017;14[cited 2018 Apr 9 ] Available from: https://www.ncbi.nlm.nih.gov/pmc/articles/PMC5702130/

20. Raspopovic S, Capogrosso M, Petrini FM, et al. Restoring natural sensory feedback in real-time bidirectional hand prostheses. Sci. Transl. Med. 2014;6(222):222ra19-222ra19.

21. Rossini PM, Micera S, Benvenuto A, et al. Double nerve intraneural interface implant on a human amputee for robotic hand control. Clin. Neurophysiol. 2010;121(5):777-783.

22. Graczyk EL, Schiefer MA, Saal HP, et al. The neural basis of perceived intensity in natural and artificial touch. Sci. Transl. Med. 2016;8(362):362ra142-362ra142.

23. Atkins DJ, Heard DC, Donovan WH. Epidemiologic overview of individuals with upper-limb loss and their reported research priorities. JPO J. Prosthet. Orthot. 1996;8(1):2-11.

24. Boretius T, Badia J, Pascual-Font A, et al. A transverse intrafascicular multichannel electrode (TIME) to interface with the peripheral nerve. Biosens. Bioelectron. 2010;26(1):62-69.

25. Oddo CM, Raspopovic S, Artoni F, et al. Intraneural stimulation elicits discrimination of textural features by artificial fingertip in intact and amputee humans [Internet]. eLife [date unknown];5[cited 2018 Apr 9 ] Available from: https:/www.ncbi.nlm.nih.gov/pmc/articles/PMC4798967/

26. Farina D, Aszmann O. Bionic Limbs: Clinical Reality and Academic Promises. Sci. Transl. Med. 2014;6(257):257ps12-257ps12.

27. Courtine G, Bloch J. Defining Ecological Strategies in Neuroprosthetics. Neuron 2015;86(1):29-33.

28. Kim LH, McLeod RS, Kiss ZHT. A new psychometric questionnaire for reporting of somatosensory percepts. J. Neural Eng. 2018;15(1):013002.

29. Simon AM, Hargrove LJ, Lock BA, Kuiken TA. A decision-based velocity ramp for minimizing the effect of misclassifications during real-time pattern recognition control. IEEE Trans. Biomed. Eng. 2011;58(8):2360-2368.

30. Humbert SD, Snyder SA, Grill WM. Evaluation of command algorithms for control of upper-extremity neural prostheses. IEEE Trans. Neural Syst. Rehabil. Eng. 2002;10(2):94-101.

31. Andreu D, Guiraud D, Souquet G. A distributed architecture for activating the peripheral nervous system. J. Neural Eng. 2009;6(2):026001.

32. Guiho T, Andreu D, López-Alvarez VM, et al. Advanced 56 Channels Stimulation System to Drive Intrafascicular Electrodes [Internet]. In: Converging Clinical and Engineering Research on Neurorehabilitation II. Springer, Cham; 2017 p. 743-747. [cited 2018 Apr 9 ] Available from: https://link.springer.com/chapter/10.1007/978-3-319-46669-9_122

33. Bisoni L, Carboni C, Raffo L, et al. An HV-CMOS Integrated Circuit for Neural Stimulation in Prosthetic Applications. IEEE Trans. Circuits Syst. II Express Briefs 2015;62(2):184-188.

34. Clemente F, D’Alonzo M, Controzzi M, et al. Non-Invasive, Temporally Discrete Feedback of Object Contact and Release Improves Grasp Control of Closed-Loop Myoelectric Transradial Prostheses. IEEE Trans. Neural Syst. Rehabil. Eng. 2016;24(12):1314-1322.

35. Mathiowetz V, Volland G, Kashman N, Weber K. Adult Norills for the Box and Block Test of Manual Dexterity. [date unknown];6.

36. Bouhassira D, Attal N, Fermanian J, et al. Development and validation of the Neuropathic Pain Symptom Inventory: Pain 2004;108(3):248-257. 
37. Ortiz-Catalan M, Guðmundsdóttir RA, Kristoffersen MB, et al. Phantom motor execution facilitated by machine learning and augmented reality as treatment for phantom limb pain: a single group, clinical trial in patients with chronic intractable phantom limb pain. The Lancet 2016;388(10062):2885-2894.

38. Carlsson AM. Assessment of chronic pain. I. Aspects of the reliability and validity of the visual analogue scale: Pain 1983;16(1):87-101.

39. Nyenhuis DL, Yamamoto C, Luchetta T, et al. Adult and geriatric normative data and validation of the profile of mood states. J. Clin. Psychol. 1999;55(1):79-86.

40. Wurth S, Capogrosso M, Raspopovic S, et al. Long-term usability and bio-integration of polyimide-based intra-neural stimulating electrodes. Biomaterials 2017;122:114-129.

41. Granata G, Iorio RD, Romanello R, et al. Phantom somatosensory evoked potentials following selective intraneural electrical stimulation in two amputees. Clin. Neurophysiol. 2018;129(6):1117-1120.

42. Jabaley ME, Wallace WH, Heckler FR. Internal topography of major nerves of the forearm and hand: a current view. J. Hand Surg. 1980;5(1):1-18.

43. Raspopovic S, Petrini FM, Zelechowski M, Valle G. Framework for the development of neuroprostheses: from basic understanding by sciatic and median nerves models to bionic legs and hands. Proc. IEEE 2017;105(1):34-49.

44. Johansson RS, Flanagan JR. Coding and use of tactile signals from the fingertips in object manipulation tasks. Nat. Rev. Neurosci. 2009;10(5):345-359.

45. Valle G, Mazzoni A, Iberite F, et al. Biomimetic Intraneural Sensory Feedback Enhances Sensation Naturalness, Tactile Sensitivity, and Manual Dexterity in a Bidirectional Prosthesis [Internet]. Neuron 2018;0(0)[cited 2018 Oct 9 ] Available from: https://www.cell.com/neuron/abstract/S08966273(18)30738-4

46. Valle G, Petrini FM, Strauss I, et al. Comparison of linear frequency and amplitude modulation for intraneural sensory feedback in bidirectional hand prostheses. Sci. Rep. 2018;8(1):16666.

47. Delgado-Martínez I, Badia J, Pascual-Font A, et al. Fascicular Topography of the Human Median Nerve for Neuroprosthetic Surgery [Internet]. Front. Neurosci. 2016;10[cited 2018 May 15 ] Available from: https://www.frontiersin.org/articles/10.3389/fnins.2016.00286/full

48. Cartwright MS, Shin HW, Passmore LV, Walker FO. Ultrasonographic Findings of the Normal Ulnar Nerve in Adults. Arch. Phys. Med. Rehabil. 2007;88(3):394-396.

49. Flor H. Phantom-limb pain: characteristics, causes, and treatment. Lancet Neurol. 2002;1(3):182-189.

50. Makin TR, Bensmaia SJ. Stability of Sensory Topographies in Adult Cortex. Trends Cogn. Sci. 2017;21(3):195-204.

51. Rognini G, Petrini FM, Raspopovic S, et al. Multisensory bionic limb to achieve prosthesis embodiment and reduce distorted phantom limb perceptions. J Neurol Neurosurg Psychiatry 2018;jnnp-2018-318570.

52. Schiefer M, Tan D, Sidek SM, Tyler DJ. Sensory feedback by peripheral nerve stimulation improves task performance in individuals with upper limb loss using a myoelectric prosthesis. J. Neural Eng. 2016;13(1):016001.

53. Graczyk EL, Resnik L, Schiefer MA, et al. Home Use of a Neural-connected Sensory Prosthesis Provides the Functional and Psychosocial Experience of Having a Hand Again [Internet]. Sci. Rep. 2018;8(1)[cited 2018 Aug 14 ] Available from: http://www.nature.com/articles/s41598-018-26952-x 


\section{Figures captions}

Fig. 1. Intraneural stimulation stabilized over time. (A) The subjects, after the implantation of four TIMEs in the median and ulnar nerves, periodically received sessions of stimulation in combination with or without the prosthesis. For the bidirectional control, the sensors readout was used to drive the intraneural stimulation. The relationship between these parameters was linear. The robotic hand was controlled through the EMG of the forearm residual muscles. The stimulation, when delivered without the prosthesis, was driven by a custom-made software through which the parameters of current were set, and the subjects' reports were recorded. The results of this characterization are shown in (B) as $75^{\text {th }}$ percentile of the phantom sensations locations evoked during the six-month clinical trial. EL1 is the electrode \#1 implanted in the proximal part of the median nerve; EL2 is electrode \#2 implanted in the distal part of the median nerve; EL3 is the one (\#3) implanted in the proximal part of the ulnar nerve; EL4 is the electrode (\#4) implanted in the distal part of the ulnar nerve. AS means active sites. Also, the number of sensations reported by ALM and LRP on the stump (the same value from FNG is negligible) over time is plotted (C), along with the stability of the sensations elicited from the phantom hand (and not the stump) of FNG, ALM, and LRP (D). The stability was evaluated in terms of location of the percept. The characterization results were used to calibrate the sensory feedback restoration system of the bidirectional prosthesis, whose motion was instead controlled by means of the electrical activity of the residual muscles of the amputated limb. (E) The types of sensations reported during the whole trial. The minimum charge needed to evoke a sensation in FNG, ALM and LRP are in (F), (G) and (H). The boxplots are computed from the sensory thresholds of 3, 3 and 4 electrodes respectively for FNG, ALM, and LRP. The averages are obtained from the sensory thresholds of the active sites, which were lower than $120 \mathrm{nC}$. Per each active site the threshold was determined as average of three repetitions. The fitting performance $\left(R^{2}\right.$ and $\left.p\right)$ of the saturating exponential function (dashed line) is reported. The function is defined as $-D+C /(B+\exp (-x / A))$. Two-tailed Friedman's test with Tukey-Kramer correction for multiple groups of data was performed.

Fig. 2. The Virtual Eggs and the Blindfolded Sensory Blocks Tests. (A) During the VET, the subjects are instructed to transfer the fragile blocks presented in front of them from one side to the other over a $15 \mathrm{~cm}$ tall wall as fast as possible and without breaking them. (B) Performance for FND $(\mathrm{N}=4 \times 2$ repetitions), ALM ( $N=27 \times 2$ repetitions) and $\operatorname{LRP}(\mathrm{N}=5 \times 2$ repetitions). The percentage of transferred blocks over the total number of blocks (transferred plus broken) with and without sensory feedback is indicated on the left. On the right, the total number of transferred blocks is displayed. Data are shown as mean values \pm SD. Statistical evaluations results in (B) were obtained using the Friedman's test. (C) Performance and total number of transferred blocks over time. The data related to the first and the last sessions, with and without sensory feedback for both subjects is reported. Statistical evaluations results were obtained using the Fisher's exact test. (D) Schematic of the BSBT experiments. (E) The performance in the BSBT for FND (N=9), ALM $(N=3)$ and LRP $(N=3)$ are shown. The score and errors were indicated, and the data are reported as mean values \pm SD. Statistical evaluations results in (E) were obtained using the two-tailed Friedman's test. (F) The BSBT performance over time is shown. The data related to the first and the last sessions, for both subjects are shown. Statistical evaluation results were obtained using the Fisher's exact test.

Fig. 3. Force Control Test. (A) Schematic of the FCT experiments. (B) The performance in the last executed FCT for FND ( $N=90)$, (C) ALM ( $N=90)$ and (D) LRP ( $N=90)$ are shown. The confusion matrices for both subjects are indicated. For FND, ALM and LRP (E) the sensor readings in the three force levels are reported as mean values \pm SD. Statistical evaluations (results in the figure) were performed using the two-tailed Friedman's test. (F) The FCT performance over time for all subjects ( $\mathrm{N}=180$ for FND, $\mathrm{N}=180$ for $\mathrm{ALM}, \mathrm{N}=180$ for LRP) is shown. Statistical evaluation results in (E) were obtained using the Fisher's exact test. 
Fig. 4. Perceived sensation levels and locations. The ranges of injected charge for each pressure level perceived by FND (A), ALM (B) and LRP (C) related to 4 active sites per subject used for the implementation of the bidirectional prosthesis are reported. Data in the figure is represented as median and $25^{\text {th }}-75^{\text {th }}$ percentiles. ${ }^{*}$ means $p<0.05$. Two-tailed Kruskal-Wallis test with Tukey-Kramer correction for multiple groups of data was performed. Sensation locations for maximum and minimum intensities reported by each patient during the modulation of charge of the stimulation train are reported (top). In the maps of hand sensations, each colored area indicates the $75^{\text {th }}$ percentile of the phantom sensation evoked for four active sites for each patient (two on median and two on the ulnar nerve). The number of statistically different pressure levels perceived by the subjects is shown in (D) as mean \pm SD.

Fig. 5. Intraneural electrodes are stable over time. The impedance of the electrodes active sites after an initial increase reached a plateau for both FNG and ALM (respectively (A) (B), top). For LRP the impedances were stable since the first week (C). The number of active sites, with impedance lower than $100 \mathrm{k} \Omega$, used to compute the average impedance is displayed for FNG, ALM and LRP (respectively (A), (B) and (C), top). Per each active site a single measure of impedance was executed. Two-tailed Friedman's test with Tukey-Kramer correction for multiple groups of data was performed. The loss of active sites (impedance higher than $100 \mathrm{k} \Omega$ ) is due to the mechanical stress related to the connection and disconnection of the electrodes cables with the external stimulator. Indeed, microscopic inspection $(C$ and $D$ ) indicates damages only at the extracorporeal transition between cables and plugs and not at the implanted transition between cables, ceramic portion and polymer-thin-film structure. The deposited thin-film electrodes adhered well on the polymer substrate and showed no signs of corrosion nor deterioration.

Fig. 6. Phantom Pain Evaluation. The NPSI, VAS and POMS-SF were recorded before the implant and during each session of neural stimulation before the explant. The NPSI evolution throughout the weeks is displayed for FND (A) and LRP (B). The VAS scores are displayed in (C) for FND and in (D) for LRP. The data are reported as mean values \pm SD. Statistical evaluations were performed between first and last session using the two-tailed Friedman's test. (E-F) The POMS-SF results related to the first (left) and the last (right) session are shown for FNG (E) and LRP (F). ${ }^{*}$ indicates a reduction from the first to the last session.

Fig. 7. FNG spontaneous phantom sensations and LRP median and ulnar nerves. (A) On the left, the localization of the not painful spontaneous phantom sensations of FNG is depicted. On the right, respectively from top to bottom, there are impact to life, and frequency occurrence evaluation questionnaires of phantom sensations filled out by FNG. (B) The picture, taken from the intraoperative microscope, shows the small size and the altered aspect of median nerve (in comparison with the adjacent ulnar nerve) of LRP.

Supp. Video 1. TIMEs implants.

Supp. Video 2. VET and BSBT tests. 